\title{
Non-Contact Characterization of Acoustoelastic Parameters of Advanced Materials by Laser-Ultrasound
}

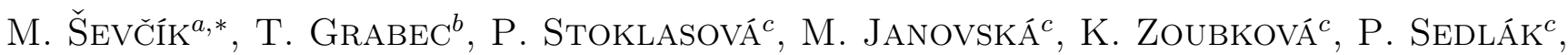 \\ H. SEINER ${ }^{c}$ AND M. LANDA ${ }^{c}$ \\ ${ }^{a}$ Faculty of Electrical Engineering, Czech Technical University in Prague, \\ Technická 2, Praha 6, 16627, Czech Republic \\ ${ }^{b}$ Nuclear Physics Institute of The Czech Academy of Science, Řež, 25068, Czech Republic \\ ${ }^{c}$ Institute of Thermomechanics of The Czech Academy of Science, \\ Dolejškova 1405/5, Praha 9, 18200, Czech Republic
}

\begin{abstract}
In advanced functional materials, where the prestress can initialize phase transitions or other structural changes, the effect of the increasing load on an acoustic wave velocity is substantial and can provide important information on the undergoing physical phenomena. In this paper, a novel method for contactless measurements of acousto-elastic parameters is presented. The contactless arrangement, based on the concept of laser-ultrasound, enables an accurate detection of small changes of the velocities of surface acoustic waves in various directions. Because of this contactless arrangement, the changes of the sample shape during the loading do not affect the results, which can be assumed as the main source of inaccuracy for classical contact methods. The experimental device and its control system is described in detail, and its application possibilities and limits are shown on examples of shape memory alloys.
\end{abstract}

DOI: 10.12693/APhysPolA.134.807

PACS/topics: 81.40.Jj, 81.70.Cv, 43.35.Yb

\section{Introduction}

Acoustoelastic effect refers to the dependence of the acoustic wave velocity on stress or strain. The interest in acoustoelasticity has been stimulated by measurements of residual stresses in polycrystalline materials [1] and the study of anharmonic behaviour of long-wavelength acoustic modes in single crystals [2].

Since the acoustoelastic coupling (stress-induced anisotropy) is very weak in structural polycrystalline materials, other factors such as temperature, microinhomogeneity, texture, which are usually neglected in engineering applications of the theory of elasticity, cannot be neglected in the study of acoustoelasticity. For this reason, the ultrasonic methods for measuring residual stresses have not been fully developed yet [3].

However, in advanced functional materials, where the prestress can initialize phase transitions or other structural changes, the evolution of acoustic wave velocity with increase of load can be substantial and can provide an important information on the undergoing physical phenomena.

The semi-linearized theory, first published by Thurston and Brugger in [4], enables to develop methods for determination of third-order elastic constants from the change of wave velocities in crystals under the influence of initial stresses. This approach was used for study of lattice sta-

*corresponding author; e-mail: sevcik@it.cas.cz bility and martensitic transformation in Cu-based shape memory alloys by Gonzalez-Comas and Manosa in [5], where complete set of third-order elastic constants of the SMAs single crystals are a function of temperature. This method for the characterization of stress-induced phase transition brings two difficulties [6]. Firstly, theory of Thurston and Brugger supposed a small difference between instantaneous and stress-free acoustic wave propagation velocities. This condition cannot be fulfilled in the case of stress-induced transformations [7]. This problem can be solved by evaluation of second-order elastic constants as a function of the applied stress. It means that in each loading step, the inverse procedure for determination of complete elastic tensor [8] will be applied, and hence softening phonon modes can be identified. Secondly, the time of flight measurement of wave velocity by the contact approach is influenced by the change of sample size and shape due to applied strain.

In this paper, both optical part and the control system of the experimental arrangement is presented. The suggested method is based on measurements of velocities of surface acoustic wave propagation along the specimen surface in various directions with respect to the loading direction. Due to the fully contactless approach, the geometrical changes of the sample do not affect the velocity measurement.

\section{Experiment}

The propagation of the planar surface acoustic waves (SAW) along the flat surface of isotropic homogeneous material exhibit minimal dispersion effect and the SAW 
velocity gives information on mechanical properties in the propagation direction. The penetration depth of the SAW corresponds to the acoustic wavelength that is large enough for the inspection of the bulk properties. Although the SAW propagation on anisotropic solids is more complex [9], the detection of the SAW propagation in various direction on the specimen surface allows to map the instantaneous anisotropic properties of tested material. The SAW propagation is constrained only on one side of the specimen and rest of the sample surface can be exposed to the external loading.

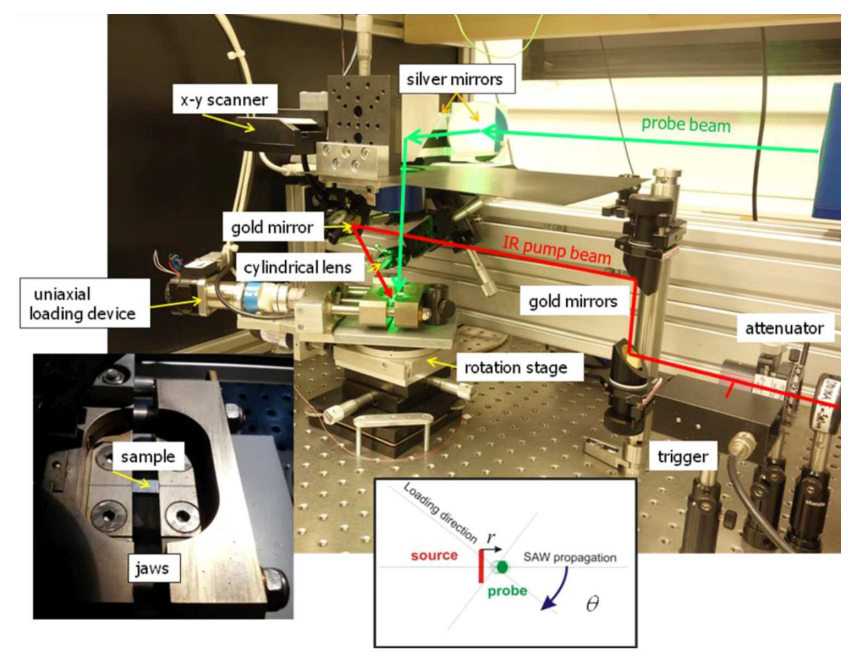

Fig. 1. The experimental setup of laser-based ultrasonic SAW method for in situ material characterization under uniaxial mechanical loading.

The applied laser technique is similar to the system described in [10]. In the presented experimental setup (Fig. 1), the SAW was excited thermoelastically by the impact of an infrared laser pulse. A beam of the Nd:YAG laser $(1064 \mathrm{~nm}$, pulse energy $>100 \mu \mathrm{J}$, pulse width $500 \mathrm{ps}$ and $1 \mathrm{kHz}$ repeating frequency) was focused via a cylindrical lens into a line-like source on the sample surface. The line source provides the generation of planar SAWs. The surface vibration was detected at variable pump-probe distances by a homodyne laser interferometer (532 nm) with an adaptive beam combiner, using two-wave mixing in a photorefractive crystal. The signal which is proportional to the instantaneous outof-plane displacement of the surface was recorded. The frequency bandwidth of the interferometer output was restricted to $100 \mathrm{MHz}$. The spot size of the probe laser beam was less than $10 \mu \mathrm{m}$. The pump-line-source spot is fixed and the point-probe spot is translated along the sample surface. This arrangement protects the sample surface from the potential damage caused by an infrared laser source. The higher level of energy density of the pump laser can cause a localized melting of the exposed surface. This effect is dependent on the properties of a tested material. The energy level is usually decreased below the melting limit and the signal-to-noise ratio of the recorded signal is attained by averaging of repeated shots in an acquisition scope card. Since the higher averaging number increases measuring time, the adjustment of the laser power and averaging number must be balanced. The averaging over 1000 shots for high speed scope card NI - PXIe 5162 10bits/5GS/s was found optimal. The optical large aperture beam (50 $\mathrm{mm}$ in diameter), outgoing from the interferometric system, is nearly collimated. Motorized translators with a pair of large silver mirrors (3 inch in diameter) and the objective lens with focus length $100 \mathrm{~mm}$ were used for precise positioning and focusing of the probe beam on the sample surface. The repeatable precision of the probe beam position is better than $0.1 \mu \mathrm{m}$. Typical translation step in the scan of pump-probe distance was $30 \mu \mathrm{m}$ in the range 200 $900 \mu \mathrm{m}$. The minimal value $(200 \mu \mathrm{m})$ of the pump-probe distance was found ideal for the elimination of the nearfield effects of the acoustic source.

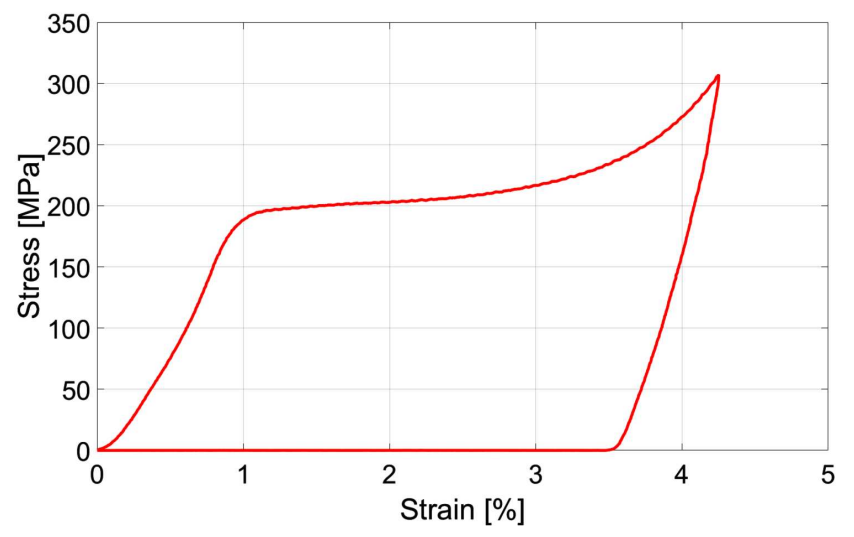

Fig. 2. Typical stress-strain dependence of stressinduced martensitic transformation in $\mathrm{Ni}-\mathrm{Ti}$.

The prismatic specimens of advanced functional materials were placed in the laboratory-made uniaxial loading two-guide-rod micro machine working in compression mode. The force was measured from strain measurement on both guide rods by the calibrated full bridge of semiconductive strain gauges with temperature compensation with precision $0.5 \mathrm{~N}$ in the range up to $6 \mathrm{kN}$. Typical stress-strain dependence of stress-induced martensitic transformation in $\mathrm{Ni}-\mathrm{Ti}$ sample can be seen in Fig. 2 . The displacement was measured by inductive transducer (LVDT) with free movable plunger (linearity better than $\pm 3 \mu \mathrm{m}$ in the range of $10 \mathrm{~mm})$. The stress-strain control of loading is available. The temperature was monitored by the miniature thermocouple $(0.3 \mathrm{~mm}$ in diameter) placed near to the specimen. The testing micromachine was rotated by the motorized rotation stage with high precision (0.3 degree).

The measuring process starts with the setting of the stress loading and then the SAW signals are recorded for each defined pump-probe distance in all given angular positions of the sample. 


\section{Results and discussion}

Typical signal data set for given loading levels and for the SAW propagation in a loading direction $($ theta $=0)$ is shown in Fig. 3. Owing to the imperfect stabilization of the interferometric setup the signal phase was changing and thus poor results were obtained after averaging. For better results, the absolute values of signals were averaged. Despite the fact that the signals in Fig. 3 are in their absolute values the arrivals of SAW and also skimming surface longitudinal waves (SSLW) are observable. A SSLW is a surface longitudinal wave that exhibits strong dispersion (with comparison to the SAW) because SSLW generates a transverse wave (TW) propagating from the surface into a bulk of the sample. Since SSLW acts as a supersonic movable source of a TW, the determination of a SSLW arrival is influenced by velocity of a transversal wave and strongly depends on spatial distribution of the finite acoustic source. Precise analysis of the SSLW waveform and evaluation of its velocity needs a more realistic model of the acoustic source and a wave propagation model. At the present time, the determined SSLW velocity can be used for the rough estimation of velocity of a longitudinal wave ( $\mathrm{LW}$ ) propagating in the bulk. By contrast to the evaluation of SSLW, the SAW velocity can be evaluated more precisely because of the
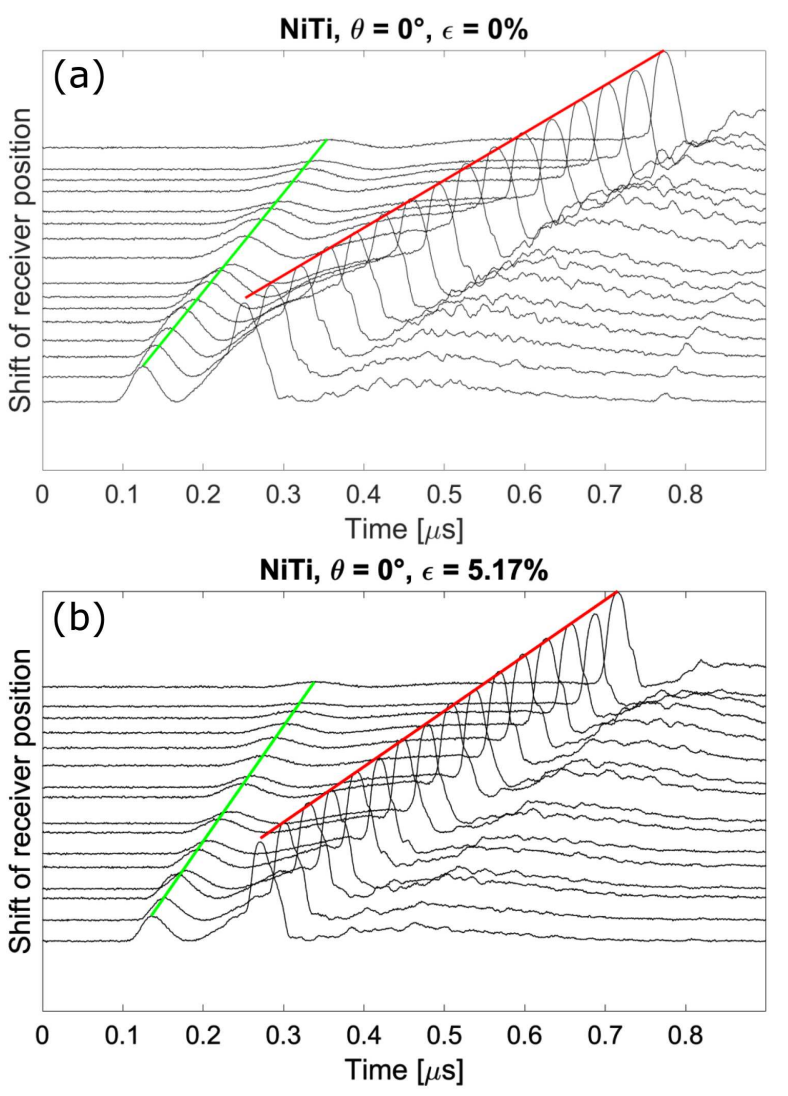

Fig. 3. Ultrasonic signal set for stress free (a) and max loaded (b) state of the $\mathrm{Ni}-49.5 \mathrm{Ti}$ sample, red $=\mathrm{SAW}$, green $=$ SSLW. perfect detectability of a wave arrival and neglectable dispersion. The presented specimen was cut from the rod of fine-grained material of $\mathrm{Ni}-49.5 \mathrm{Ti}(50.5$ at. $\% \mathrm{Ni}-$ 49.5 at.\% $\mathrm{Ti}$ ), for details see [11].

Evolution of SAW velocities at various directions of propagation starts from common origin at stress-free state (Fig. 4). The SAW at the compression direction rapidly increases its velocity from $0.5 \%$ of strain whereas SAW velocities decrease with increase of the propagation angle. Minimal value of velocity is at perpendicular direction to the loading. This anisotropization can be explained by elasticity softening at R-phase transformation [11]. Following martensitic transformation and martensitic reorientation causes increase of the SAW velocity. During unloading of the final martensite, the SAW velocity decreases along the loading curve without hysteresis.

Detailed context with microstructural processes is possible to discuss after evaluation of elastic properties by inversion of acoustic data in [8]. In this paper, the described example illustrates potential of the laser-based ultrasonic method for in situ material characterization during mechanical testing.

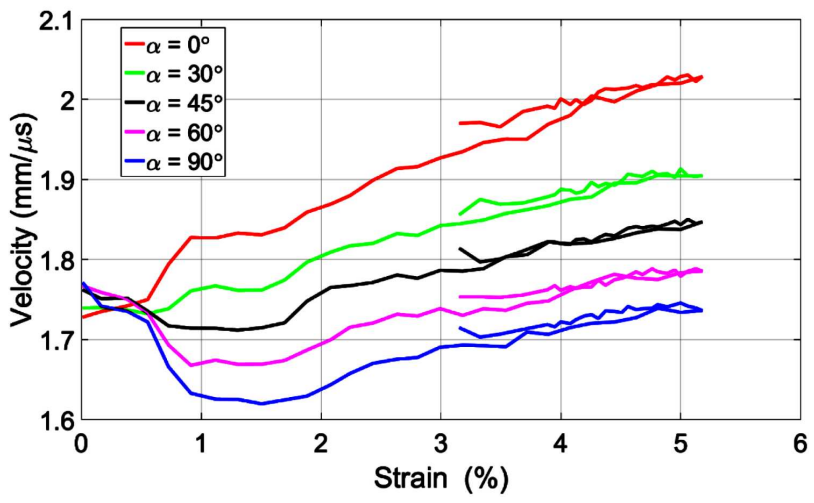

Fig. 4. SAWs velocities different directions with respect to loading.

\section{Conclusion}

A novel method for acoustoelastic characterization of functional materials was presented. The contactless arrangement, based on the concept of laser-ultrasound, enables accurate detection of small changes of the surface velocities and estimation of bulk acoustic waves in various directions on a small sample loaded uniaxially in a testing machine. Due to this arrangement, the changes of the shape of the sample during the loading do not affect the results, which can be assumed as the main source of inaccuracy for classical contact methods. The experimental device and its control system was described in detail, and its applicability was shown on $\mathrm{Ni}-49.5 \mathrm{Ti}$ sample. The described experimental technique has a great potential for in situ non-destructive characterization of microstructural processes during material testing. 


\section{Acknowledgments}

This work has been supported by the project of the Czech Science Foundation (17-04871S), and by OP RDE, MEYS, under the project ESS - SCANDINAVIA - CZ — PROJECT CZ.02.1.01/0.0/0.0/16_013/0001794.

\section{References}

[1] Y.H. Pao, W. Sachse, H. Fukuoka, in: Physical Acoustics, Vol. XVII, Eds. W.P. Mason, R.N. Thurston, Academic Press, London 1984, p. 62.

[2] M.A. Breazeale, J. Philip, in Ref. [1], p. 2.

[3] E. Schneider, in: Structural and Residual Stress Analysis by Non-Destructive Methods, Ed. V. Hauk, Elsevier Sci. B, Amsterdam 1997.

[4] R.N. Thurston, K. Brugger, Phys. Rev. 135, A1604 (1964).
[5] A. Gonzales-Comas, Ll. Manosa, Philos. Mag. A 80 , 1681 (2009)

[6] T. Černoch, M. Landa, V. Novák, P. Sedlák, P. Šittner, J. Alloys Comp. 378, 140 (2004).

[7] H. Seiner, P. Stoklasová, P. Sedlák, M. Ševčík, M. Janovská, M. Landa, T. Fukuda, T. Yamaguchi, T. Kakeshita, Acta Mater. 105, 182 (2016).

[8] T. Grabec, K. Zoubková, P. Stoklasová, M. Ševčík, P. Sedlák, M. Janovská, H. Seiner, M. Landa, Acta Phys. Pol. A 134, 811 (2018).

[9] D. Royer, E. Dieulesaint, J. Acoust. Soc. Am. $\mathbf{7 6}$ 1438 (1984).

[10] P. Stoklasová, P. Sedlák, H. Seiner, M. Landa, Ultrasonics 56, 381 (2015).

[11] M. Thomasová, H. Seiner, P. Sedlák, M. Frost, M. Ševčík, I. Szurman, R. Kocich, J. Drahokoupil, P. Sittner, M. Landa, Acta Mater. 123, 146 (2017). 